\title{
Review of: "Length of stay following percutaneous left atrial appendage occlusion: Data from the prospective, multicenter Amplatzer Amulet Occluder Observational Study"
}

\author{
Jacques Mansourati ${ }^{1}$ \\ 1 Centre Hospitalier Universitaire de Brest
}

Potential competing interests: Consultancy: ABBOTT and Boston Scientific

This paper gives a detailed description of adverse events occurring in a population of more than 1000 patients receiving the Amplatzer Amulet Occluder. Although not randomized, it is a real life evaluation with interesting results. The main limitation is reported by the authors: as it is a Multicenter trial, discharge policies are not the same in all centers. However, it doesn't affect prolonged in-hospital stay but only short length stay.

Tables and figures are well presented.

Although the results were predictable, the main interest of the paper is the description of different outcomes of patients.

Therefore, this paper is an interesting report in this field. 